\title{
Comparative Analysis of Backcross and Selfed Progenies in Sunflower (Helianthus annuus L.)
}

\author{
Sagar Iliger $^{1 *}$, V. V. Kulkarni ${ }^{2}$, J. R. Diwan ${ }^{1}$, S. Muniswami ${ }^{3}$ and S. B. Mallesh ${ }^{4}$
}

${ }^{1}$ Department of Genetics and Plant Breeding, ${ }^{2}$ Main Agricultural Research Station, ${ }^{3}$ Zonal Agricultural Research Station Kalaburagi, ${ }_{4}^{4}$ Department of Plant Pathology, ARS Hagari, University of Agricultural Sciences, Raichur- 584 104, (Karnataka) India

*Corresponding author

\section{A B S T R A C T}

\begin{tabular}{l} 
K e y w o r d s \\
$\begin{array}{l}\text { Sunflower, Wide } \\
\text { hybridization, } \\
\text { Backcrossing, } \\
\text { Selfing, Pre- } \\
\text { breeding }\end{array}$ \\
\hline Article Info \\
$\begin{array}{l}\text { Accepted: } \\
\text { 12 August } 2020 \\
\text { Available Online: } \\
\text { 10 September } 2020\end{array}$ \\
\hline
\end{tabular}

\section{Keywords}

Sunflower, Wide (a)

\section{Introduction}

Sunflower(Helianthus annuusL.) is one of the important oilseed crops of India. Sunflower production continues to face challenges from both abiotic and biotic factors as well as from today's ever-changing market needs. However, the limited genetic variability in cultivated sunflower has slowed the future improvement of the crop and has placed the crop in a vulnerable position. The overexploitation of a single CMS PET1 cytoplasm and a few fertility restoration genes for worldwide sunflower production makes the crop extremely vulnerable with narrow genetic base. Diversity in CMS base and resistance to various diseases is strategically needed. Evaluation of wild species has provided information about useful genes for future sunflower improvement. However, there are still numerous genes in wild sunflower species yet to be identified and introgressed into cultivated sunflower (Jan et al., 2006). Though crop improvement in sunflower has resulted in the development of many promising hybrids and populations, 
many biotic and abiotic stresses are still limiting the productivity. The assessment of per se utility of interspecific derived germplasm for their use in commercial plant breeding programs is an important prerequisite.

There is a need to broaden the genetic base of cultivar germplasm, break the yield stagnation, development of material for diverse location and situations and also for the introgression of specific characters from wild Helianthus species to cultivated species through pre-breeding programme. The backcross-derived inbred lines variability in terms of several distinct phenotypic characters not present in the cultivated species as a source of maintainer or restorers for different characters. (Sujatha et al., 2008). The process of creating variability in desirable direction can be achieved through wide hybridization and further selection of introgressed lines. The variability can be created by following several breeding methods viz., hybridization, introduction, backcrossing and inter crossing between different species and genera. The present work was planned to compare and identify best breeding method i.e. whether backcrossing or selfing is prominent in generation advancement to create higher variability in desirable direction in pre-bred material of sunflower.

\section{Materials and Methods}

The experiment was conducted during late Kharif 2018 at Main Agriculture Research Station, University of Agricultural Sciences, Raichur. The experimental material consisted of 24 introgressed family progenies of backcross and selfed generation along with parental CMS lines in which each entry was sown in two rows of $4 \mathrm{~m}$ length with spacing of $60 \times 30 \mathrm{~cm}$ introgressed families derived by crossing four wild accessions of $H$. annuus (ANN-61, ANN-98, ANN-19 and ANN-114) and three CMS lines (CMS 103B, CMS 104B and $\mathrm{CMS} 38 \mathrm{~B}$ ). Further $\mathrm{BC}_{2} \mathrm{~F}_{3}$ generation was derived by backcrossing individual plants of $\mathrm{BC}_{1} \mathrm{~F}_{3}$ to its respective cultivated genotype whereas the individual plants grown in $\mathrm{BC}_{1} \mathrm{~F}_{3}$ were selfed to generate $\mathrm{BC}_{1} \mathrm{~F}_{4}$. In further generation also while deriving backcross and selfed generation individual plant identity of different wild accession was maintained.

In the present investigation observations were recorded and analysed for eleven traits viz., number of leaves, plant height, head diameter, stem girth, test weight, seed yield per plant, volume weight, leaf length, days to 50 per cent flowering, days to maturity, oil content (\%).The data recorded were processed with statistical parameters viz., range, mean, standard error and coefficient of variation for all traits. The data was subjected to F-test or One way ANOVA to know the variability between the families and within the families of different crosses of backcross and selfed generation.

\section{Results and Discussion}

In present investigation, one way ANOVA was used to know the between family variance and within family variance for each trait with different backcross and selfed progenies. Four types of comparison were done for variance analysis. In first comparison backcross and selfed progenies were separately analyzed for each trait and each cross. While in second comparison backcross and selfed progenies were analyzed for each trait to know the variance between backcross and selfed progenies of each cross. Whereas, in third comparison, whole backcross $\left(\mathrm{BC}_{2} \mathrm{~F}_{3}\right)$ and selfed $\left(\mathrm{BC}_{1} \mathrm{~F}_{4}\right)$ progenies were analyzed separately to know variance among different crosses. In fourth comparison total population variance (includes both backcross and selfed progenies) was analyzed across different crosses. 
In first comparison, significant difference was observed for both between family variance and within family variance of $103 \mathrm{~B}$ derived backcrosses and selfed progenies for the traits such as number of leaves, plant height, leaf length and yield. However, some of the traits were shown significant difference only for selfed progenies of $103 \mathrm{~B}$ crosses viz., head diameter, stem diameter and test weight. While in 104B crosses, plant height, leaf length and seed yield were recorded significant difference for both between family variance and within family variance of backcross and selfed progenies. Traits like number of leaves, stem diameter and test weight were showed significant difference only in selfed progenies of 104B crosses. Whereas in $38 \mathrm{~B}$ crosses head diameter is the only trait showed significant difference of variance in both backcross and selfed progenies.

However, four traits were shown significant difference of variance only in selfed progenies of 38B cross viz., number of leaves, plant height, test weight and seed yield. Leaf length is the only character which expressed significant difference of variance in backcross progenies of $38 \mathrm{~B}$ crosses (Table 1).

In second comparison, both backcross and selfed progenies of each cross were analyzed to know variance of between family and within family variance. In 103B crosses, significant difference was observed for all the traits except leaf length i.e. there was no difference existing between backcross and selfed progenies of 103B crosses of Leaf length. While in 104B crosses also all traits were shown significant difference backcross and selfed progenies except stem diameter. Whereas in 38B crosses number of leaves, head diameter, plant height, days to 50 per cent flowering, days to maturity, test weight, volume weight, yield and oil content were revealed significant difference between backcross and selfed progenies except stem diameter and leaf length (Table 2).

In third comparison, whole backcross $\left(\mathrm{BC}_{2} \mathrm{~F}_{3}\right)$ and selfed $\left(\mathrm{BC}_{1} \mathrm{~F}_{4}\right)$ progenies were analyzed separately to know the variance between crosses and within crosses. In backcross progenies all the traits were shown significant difference except oil content ie., No difference was observed for oil content trait in backcross progenies across different crosses. While in selfed progenies head diameter, stem diameter and test weight were recorded significant difference of variance and rest of the characters were shown non significance (Table 3).

In fourth comparison, total population variance (includes both backcross and selfed progenies) was analyzed among different crosses. Traits such as head diameter, stem diameter, test weight, volume weight and yield were revealed significant difference of variance among different crosses irrespective of backcross and selfed progenies. Remaining all traits were shown non-significant variance (Table 4).

A comparative summary of means and ranges of yield and yield attributing traits across different crosses of backcross and selfed progenies summarized in table 5 .

Head diameter in cultivated sunflower produces larger head compared to wild annual diploid $H$. argophyllus (Vishnutej et al., 2016). The cultivated lines had average head diameter was $14.4 \mathrm{~cm}$. The backcross progenies showed head diameter of 12.7-15.8 $\mathrm{cm}$ Indicating dominance of parental CMS lines whereas selfed progenies recorded intermediate $(8.5-10.4 \mathrm{~cm})$ head diameter. Variability observed for head diameter was more in backcross progenies of 103B, 104B, 38B crosses as compared to their selfed progenies. 
Table.1 Analysis of variance for between and within families of backcross and selfed progenies derived from 103B, 104B and 38B in Sunflower

\begin{tabular}{|c|c|c|c|c|c|c|c|c|c|c|c|c|}
\hline \multirow{2}{*}{\multicolumn{2}{|c|}{ Source of variation }} & \multirow[b]{2}{*}{ df } & \multicolumn{2}{|c|}{$\begin{array}{l}\text { No of leaves } \\
\text { (MSS) }\end{array}$} & \multicolumn{2}{|c|}{$\begin{array}{l}\text { Plant height } \\
\text { (MSS) }\end{array}$} & \multicolumn{2}{|c|}{$\begin{array}{l}\text { Head diameter } \\
\text { (MSS) }\end{array}$} & \multicolumn{2}{|c|}{$\begin{array}{l}\text { Stem diameter } \\
\text { (MSS) }\end{array}$} & \multicolumn{2}{|c|}{$\begin{array}{l}\text { Test weight } \\
\text { (MSS) }\end{array}$} \\
\hline & & & BC & Self & BC & Self & BC & Self & BC & Self & BC & Self \\
\hline \multirow[t]{2}{*}{ 103B } & $\begin{array}{l}\text { Between } \\
\text { families }\end{array}$ & 7 & $159.48^{*}$ & $1860.8^{* *}$ & $942.65^{* *}$ & $3301.73 * *$ & 17.00 & $18.29 * *$ & 3.18 & $9.27 * *$ & 3.11 & $2.77 * *$ \\
\hline & $\begin{array}{l}\text { Within } \\
\text { families }\end{array}$ & 32 & $53.67^{*}$ & $313.21 * *$ & $116.40 * *$ & $377.40 * *$ & 7.66 & $3.55^{* *}$ & 1.14 & $0.59 * *$ & 1.56 & $0.73 * *$ \\
\hline \multirow[t]{2}{*}{ 104B } & $\begin{array}{l}\text { Between } \\
\text { families }\end{array}$ & 7 & 96.32 & 981.36* & $1952.5^{* *}$ & $3070.86 * *$ & 5.85 & 4.30 & $6.00 * *$ & 2.37 & 1.98 & $2.77 *$ \\
\hline & $\begin{array}{l}\text { Within } \\
\text { families }\end{array}$ & 32 & 49.91 & $314.7 *$ & $272.18 * *$ & $311.09 * *$ & 5.85 & 2.86 & $1.55^{* *}$ & 1.22 & 1.25 & $0.92 *$ \\
\hline \multirow[t]{2}{*}{ 38B } & $\begin{array}{l}\text { Between } \\
\text { families }\end{array}$ & 7 & 44.22 & $1706.04 * *$ & 464.91 & $2601.42 * *$ & $16.32 * *$ & $68.54 * *$ & 1.34 & 3.06 & 2.20 & $2.76^{* *}$ \\
\hline & $\begin{array}{l}\text { Within } \\
\text { families }\end{array}$ & 32 & 26.82 & $228.65^{* *}$ & 313.12 & $323.56^{* *}$ & $3.92 * *$ & $4.40 * *$ & 1.27 & 2.17 & 1.85 & $0.79 * *$ \\
\hline
\end{tabular}

$*=5$ per cent level of significance $* *=1$ per cent level of significance

Contd...

\begin{tabular}{|c|c|c|c|c|c|c|c|c|c|c|}
\hline \multirow{2}{*}{\multicolumn{2}{|c|}{ Source of variation }} & \multirow[b]{2}{*}{ df } & \multicolumn{2}{|c|}{$\begin{array}{l}\text { Leaf length } \\
\text { (MSS) }\end{array}$} & \multicolumn{2}{|c|}{$\begin{array}{c}\text { Volume weight } \\
\text { (MSS) }\end{array}$} & \multicolumn{2}{|c|}{$\begin{array}{l}\text { Yield } \\
\text { (MSS) }\end{array}$} & \multicolumn{2}{|c|}{$\begin{array}{l}\text { Oil content } \\
\text { (MSS) }\end{array}$} \\
\hline & & & BC & Self & BC & Self & BC & Self & BC & Self \\
\hline \multirow[t]{2}{*}{ 103B } & $\begin{array}{l}\text { Between } \\
\text { Families }\end{array}$ & 7 & $35.80 * *$ & $41.49 * *$ & 1.29 & 0.46 & $184.68 * *$ & $144.68 * *$ & 16.44 & 8.90 \\
\hline & $\begin{array}{l}\text { Within } \\
\text { Families }\end{array}$ & 32 & $9.42 * *$ & $8.69 * *$ & 0.79 & 0.26 & $42.57 * *$ & $21.49 * *$ & 13.49 & 8.90 \\
\hline \multirow[t]{2}{*}{ 104B } & $\begin{array}{l}\text { Between } \\
\text { Families }\end{array}$ & 7 & $49.44 * *$ & $50.45^{* *}$ & 1.06 & 0.50 & $89.58 * *$ & $31.11^{*}$ & 15.37 & 7.71 \\
\hline & $\begin{array}{l}\text { Within } \\
\text { Families }\end{array}$ & 32 & $8.97 * *$ & $5.51 * *$ & 0.95 & 0.45 & $22.75 * *$ & $10.87 *$ & 9.70 & 4.45 \\
\hline \multirow[t]{2}{*}{$38 B$} & $\begin{array}{l}\text { Between } \\
\text { Families }\end{array}$ & 7 & $24.97 *$ & 22.57 & 2.36 & 0.56 & 73.45 & $79.55 * *$ & 11.77 & 5.44 \\
\hline & $\begin{array}{l}\text { Within } \\
\text { Families }\end{array}$ & 32 & $9.03 *$ & 13.28 & 1.17 & 0.33 & 36.87 & $14.11 * *$ & 10.38 & 2.68 \\
\hline
\end{tabular}

$*=5$ per cent level of significance $* *=1$ per cent level of significance 
Table.2 Analysis of variance backcross Vs selfed progenies of Sunflower

\begin{tabular}{|c|c|c|c|c|c|c|c|c|}
\hline \multirow{2}{*}{\multicolumn{2}{|c|}{ Source of variation }} & & $\begin{array}{l}\text { No of leaves } \\
\text { (MSS) }\end{array}$ & $\begin{array}{l}\text { Plant height } \\
\text { (MSS) }\end{array}$ & $\begin{array}{c}\text { Head diameter } \\
\text { (MSS) }\end{array}$ & $\begin{array}{l}\text { Stem diameter } \\
\text { (MSS) }\end{array}$ & $\begin{array}{c}\text { DFF } \\
\text { (MSS) }\end{array}$ & $\begin{array}{c}\text { DM } \\
\text { (MSS) }\end{array}$ \\
\hline & & df & BC vs Self & BC vs Self & BC vs Self & BC vs Self & BC vs Self & BC vs Self \\
\hline \multirow[b]{2}{*}{ 103B } & $\begin{array}{c}\text { Between } \mathrm{BC} \text { and self } \\
\text { generation }\end{array}$ & 1 & $17052.8 * *$ & $4708.61 * *$ & $1058.54 * *$ & $32.00 * *$ & $729.00 * *$ & $1332.25^{* *}$ \\
\hline & $\begin{array}{c}\text { Within } \mathrm{BC} \text { and self } \\
\text { generation }\end{array}$ & 78 & $331.82 * *$ & $583.49 * *$ & $7.77 * *$ & $1.83 * *$ & $8.05^{* *}$ & $15.55 * *$ \\
\hline \multirow[b]{2}{*}{ 104B } & $\begin{array}{c}\text { Between } \mathrm{BC} \text { and self } \\
\text { generation }\end{array}$ & 1 & $6462.01 * *$ & $41961.96 * *$ & $784.37 * *$ & 0.68 & $248.06 * *$ & $529.00 * *$ \\
\hline & $\begin{array}{c}\text { Within } \mathrm{BC} \text { and self } \\
\text { generation }\end{array}$ & 78 & $246.30 * *$ & $690.10 * *$ & $4.49 * *$ & 1.89 & $4.52 * *$ & $23.07 * *$ \\
\hline \multirow[b]{2}{*}{ 38B } & $\begin{array}{c}\text { Between } \mathrm{BC} \text { and self } \\
\text { generation }\end{array}$ & 1 & $6037.81 * *$ & $27937.81 * *$ & $651.11 * *$ & 2.01 & $333.06 * *$ & $132.25^{*}$ \\
\hline & $\begin{array}{c}\text { Within } \mathrm{BC} \text { and self } \\
\text { generation }\end{array}$ & 78 & $261.88 * *$ & $536.38 * *$ & $11.03 * *$ & 1.81 & $5.49 * *$ & $15.53 *$ \\
\hline
\end{tabular}

Contd...

\begin{tabular}{|c|c|c|c|c|c|c|c|}
\hline & \multirow[t]{2}{*}{ Source of variation } & \multirow[b]{2}{*}{ df } & \multirow{2}{*}{$\begin{array}{c}\text { Leaf length } \\
\text { (MSS) } \\
\text { BC vs Self }\end{array}$} & \multirow{2}{*}{$\begin{array}{c}\text { Test weight } \\
\text { (MSS) } \\
\text { BC vs Self }\end{array}$} & \multirow{2}{*}{$\begin{array}{c}\begin{array}{c}\text { Volume weight } \\
\text { (MSS) }\end{array} \\
\text { BC vs Self }\end{array}$} & \multirow{2}{*}{$\begin{array}{c}\text { Yield } \\
\text { (MSS) } \\
\text { BC vs Self }\end{array}$} & \multirow{2}{*}{$\begin{array}{l}\text { Oil content } \\
\text { (MSS) } \\
\text { BC vs Self }\end{array}$} \\
\hline & & & & & & & \\
\hline \multirow[b]{2}{*}{ 103B } & $\begin{array}{c}\text { Between } \mathrm{BC} \text { and self } \\
\text { generation }\end{array}$ & 1 & 22.36 & $38.64 * *$ & $5.832 * *$ & $1122.38 * *$ & $47.95^{*}$ \\
\hline & $\begin{array}{c}\text { Within } \mathrm{BC} \text { and self } \\
\text { generation }\end{array}$ & 78 & 14.37 & $1.47 * *$ & $0.59 * *$ & $55.84 * *$ & $740.74 *$ \\
\hline \multirow[b]{2}{*}{ 104B } & $\begin{array}{c}\text { Between } \mathrm{BC} \text { and self } \\
\text { generation }\end{array}$ & 1 & $139.12 * *$ & $38.22 * *$ & $5.85^{* *}$ & $447.97 * *$ & $100.68 * *$ \\
\hline & $\begin{array}{c}\text { Within } \mathrm{BC} \text { and self } \\
\text { generation }\end{array}$ & 78 & $14.90 * *$ & $1.32 * *$ & $0.71 * *$ & $24.62 * *$ & $7.87 * *$ \\
\hline \multirow[b]{2}{*}{ 38B } & $\begin{array}{c}\text { Between } \mathrm{BC} \text { and self } \\
\text { generation }\end{array}$ & 1 & 10.22 & $15.75 * *$ & $5.75^{*}$ & $2471.97 * *$ & $139.92 * *$ \\
\hline & $\begin{array}{c}\text { Within BC and self } \\
\text { generation }\end{array}$ & 78 & 13.42 & $1.53 * *$ & $0.88 *$ & $34.64 * *$ & $6.90 * *$ \\
\hline
\end{tabular}

$*=5$ per cent level of significance $* *=1$ per cent level of significance 
Table.3 Analysis of variance between and within crosses for backcross and selfed progenies in Sunflower

\begin{tabular}{|c|c|c|c|c|c|c|c|c|}
\hline \multicolumn{2}{|c|}{ Source of variation } & \multirow{2}{*}{\begin{tabular}{r|}
$\mathbf{d f}$ \\
2
\end{tabular}} & \multirow{2}{*}{$\begin{array}{c}\begin{array}{c}\text { No of leaves } \\
\text { (MSS) }\end{array} \\
180.92^{*}\end{array}$} & \multirow{2}{*}{$\begin{array}{c}\text { Plant height } \\
\text { (MSS) } \\
3204.74 * *\end{array}$} & \multirow{2}{*}{$\begin{array}{c}\text { Head diameter } \\
\text { (MSS) } \\
139.02 * *\end{array}$} & \multirow{2}{*}{$\begin{array}{c}\text { Stem diameter } \\
\text { (MSS) } \\
30.90^{* *}\end{array}$} & \multirow{2}{*}{$\begin{array}{c}\begin{array}{c}\text { DFF } \\
\text { (MSS) }\end{array} \\
85.16^{* *}\end{array}$} & \multirow{2}{*}{$\begin{array}{c}\begin{array}{c}\text { DM } \\
\text { (MSS) }\end{array} \\
124.04 * *\end{array}$} \\
\hline Backcross & Between crosses & & & & & & & \\
\hline $\begin{array}{c}\text { generation } \\
\left(\mathbf{B C}_{2} \mathbf{F}_{3}\right)\end{array}$ & Within cross & 117 & $53.6^{*}$ & $392.95 * *$ & $7.11 * *$ & $1.97 * *$ & $5.31 * *$ & $14.13 * *$ \\
\hline \multirow{2}{*}{$\begin{array}{c}\text { Selfed } \\
\text { generation } \\
\left(\mathbf{B C}_{1} \mathbf{F}_{4}\right)\end{array}$} & Between crosses & 2 & 648.67 & 2188.58 & $154.53 * *$ & $20.88 * *$ & 0.79 & 43.62 \\
\hline & Within cross & 117 & 486.56 & 813.70 & $8.41 * *$ & $1.96 * *$ & 6.73 & 21.97 \\
\hline
\end{tabular}

\begin{tabular}{|c|c|c|c|c|c|c|c|}
\hline \multicolumn{2}{|c|}{ Source of variation } & df & $\begin{array}{c}\text { Leaf length } \\
(\mathbf{M S S})\end{array}$ & $\begin{array}{c}\text { Test weight } \\
(\mathbf{M S S})\end{array}$ & $\begin{array}{c}\text { Volume weight } \\
\text { (MSS) }\end{array}$ & $\begin{array}{c}\text { Yield } \\
(\mathbf{M S S})\end{array}$ & $\begin{array}{c}\text { Oil content } \\
(\mathbf{M S S})\end{array}$ \\
\hline $\begin{array}{c}\text { Backcross } \\
\text { generation } \\
\left(\mathbf{B C}_{\mathbf{2}} \mathbf{F}_{\mathbf{3}}\right)\end{array}$ & Between crosses & 2 & $70.91^{* *}$ & $9.10^{* *}$ & $11.56^{* *}$ & $526.10^{* *}$ \\
\hline $\begin{array}{c}\text { Selfed generation } \\
\left(\mathbf{B C}_{\mathbf{1}} \mathbf{F}_{\mathbf{4}}\right)\end{array}$ & Within cross & 117 & $14.09^{* *}$ & $1.41^{* *}$ & $0.94^{* *}$ & $48.75^{* *}$ & 11.79 \\
\hline
\end{tabular}

$*=5$ per cent level of significance $* *=1$ per cent level of significance

Table.4 Analysis of segregating progenies derived from backcrossing and selfing in Sunflower

\begin{tabular}{|c|c|c|c|c|c|c|c|c|}
\hline \multicolumn{2}{|c|}{ Source of variation } & \multirow{2}{*}{ df } & \multirow{2}{*}{$\begin{array}{c}\text { No of leaves } \\
\text { (MSS) } \\
\text { BC + self }\end{array}$} & \multirow{2}{*}{$\begin{array}{c}\text { Plant height } \\
\text { (MSS) } \\
\text { BC + self }\end{array}$} & \multirow{2}{*}{$\begin{array}{c}\text { Head diameter } \\
\text { (MSS) } \\
\text { BC + self }\end{array}$} & \multirow{2}{*}{$\begin{array}{c}\text { Stem diameter } \\
\text { (MSS) } \\
\text { BC + self }\end{array}$} & \multirow{2}{*}{$\begin{array}{c}\text { DFF } \\
(\text { MSS }) \\
\text { BC + self }\end{array}$} & \multirow{2}{*}{$\begin{array}{c}\text { DM } \\
\text { (MSS) } \\
\text { BC + self }\end{array}$} \\
\hline & & & & & & & & \\
\hline Both backcross & Between crosses & 2 & 298.58 & 445.56 & $280.92 * *$ & $20.88^{* *}$ & 51.06 & 11.08 \\
\hline $\begin{array}{l}\text { and selfed } \\
\text { generation }\end{array}$ & Within cross & 237 & 398.77 & 910.49 & $18.19 * *$ & $1.96 * *$ & 34.73 & 61.15 \\
\hline
\end{tabular}

\begin{tabular}{|c|c|c|c|c|c|c|c|}
\hline \multicolumn{2}{|c|}{ Source of variation } & \multirow[b]{2}{*}{ df } & \multirow{2}{*}{$\begin{array}{c}\text { Leaf length } \\
\text { (MSS) } \\
\text { BC + self }\end{array}$} & \multirow{2}{*}{$\begin{array}{c}\text { Test weight } \\
\text { (MSS) } \\
\text { BC+ self }\end{array}$} & \multirow{2}{*}{$\begin{array}{c}\text { Volume weight } \\
\text { (MSS) } \\
\text { BC + self }\end{array}$} & \multirow{2}{*}{$\begin{array}{c}\text { Yield } \\
\text { (MSS) } \\
\text { BC+ self }\end{array}$} & \multirow{2}{*}{$\begin{array}{c}\text { Oil content } \\
\text { (MSS) } \\
\text { BC + self }\end{array}$} \\
\hline & & & & & & & \\
\hline \multirow{2}{*}{$\begin{array}{l}\text { Both backcross and } \\
\text { selfed generation }\end{array}$} & Between crosses & 2 & 36.00 & $9.56 * *$ & $5.26^{* *}$ & $395.06 * *$ & 4.11 \\
\hline & Within cross & 237 & 14.77 & $1.81 * *$ & $0.79 * *$ & $54.94 * *$ & 9.21 \\
\hline
\end{tabular}

$*=5$ per cent level of significance $* *=1$ per cent level of significance 
Table.5 Mean per se performance of parents and crosses for different characters of backcross and selfed progenies of Sunflower

\begin{tabular}{|c|c|c|c|c|c|c|}
\hline \multirow[t]{2}{*}{ Traits } & \multirow[t]{2}{*}{ Crosses } & \multirow{2}{*}{$\begin{array}{c}\text { Parents } \\
\text { Mean }\end{array}$} & \multicolumn{2}{|c|}{$\begin{array}{l}\text { Backcross progenies } \\
\left(\mathrm{BC}_{2} \mathrm{~F}_{3}\right)\end{array}$} & \multicolumn{2}{|c|}{ Selfed progenies $\left(\mathrm{BC}_{1} \mathrm{~F}_{4}\right.$} \\
\hline & & & Range & Mean & Range & Mean \\
\hline \multirow[t]{3}{*}{ Number of leaves } & 103B & 24 & $22-58$ & 36 & $22-114$ & 66 \\
\hline & 104B & 30 & $27-56$ & 41 & $26-116$ & 59 \\
\hline & $38 \mathrm{~B}$ & 34 & $28-53$ & 38 & 14-95 & 56 \\
\hline \multirow[t]{3}{*}{ Plant height } & 103B & 89 & $105-175$ & 138.5 & $61.5-170$ & 123.2 \\
\hline & 104B & 118 & $105-200$ & 154.2 & $62-163$ & 108.4 \\
\hline & $38 \mathrm{~B}$ & 117 & $120-190$ & 108.4 & $72-172$ & 116.5 \\
\hline \multirow[t]{3}{*}{ Head diameter } & 103B & 14.2 & $11-25$ & 15.8 & $4-14.3$ & 8.5 \\
\hline & 104B & 14.2 & $9-18.5$ & 12.7 & $3.3-11.6$ & 6.5 \\
\hline & $38 \mathrm{~B}$ & 15 & $11-21$ & 16.3 & $4-18.5$ & 10.4 \\
\hline \multirow[t]{3}{*}{ Stem diameter } & 103B & 6.2 & $4.4-9.5$ & 6.5 & $4.1-9.8$ & 7.8 \\
\hline & 104B & 5.3 & $3.5-11$ & 6.3 & $4.1-8.6$ & 6.1 \\
\hline & $38 \mathrm{~B}$ & 5.5 & $4.2-9.2$ & 6.4 & $4.2-11.5$ & 6.7 \\
\hline
\end{tabular}

\begin{tabular}{|c|c|c|c|c|c|c|}
\hline \multirow[t]{2}{*}{ Traits } & \multirow[t]{2}{*}{ Crosses } & \multirow{2}{*}{$\begin{array}{c}\text { Parents } \\
\text { Mean }\end{array}$} & \multicolumn{2}{|c|}{$\begin{array}{c}\text { Backcross progenies } \\
\left(\mathrm{BC}_{2} \mathrm{~F}_{3}\right)\end{array}$} & \multicolumn{2}{|c|}{ Selfed progenies $\left(\mathrm{BC}_{1} \mathrm{~F}_{4}\right)$} \\
\hline & & & Range & Mean & Range & Mean \\
\hline \multirow[t]{3}{*}{ Test weight } & 103B & 3.17 & $2-7$ & 4.10 & $1.2-5.3$ & 2.96 \\
\hline & 104B & 3.78 & $2.3-6.4$ & 4.13 & $1.1-5.1$ & 2.75 \\
\hline & $38 \mathrm{~B}$ & 5.87 & $2.3-7$ & 4.56 & $1.4-5.8$ & 3.67 \\
\hline \multirow{3}{*}{$\begin{array}{c}\text { Seed yield per } \\
\text { plant }\end{array}$} & 103B & 14.28 & $16-41.6$ & 21.2 & $5-30.8$ & 13.7 \\
\hline & 104B & 14.75 & $6.7-30.8$ & 15.8 & $5-20.4$ & 11.1 \\
\hline & $38 \mathrm{~B}$ & 20.15 & $9.25-32.8$ & 22.8 & $5.7-29.8$ & 11.6 \\
\hline \multirow[t]{3}{*}{ Leaf length } & 103B & 15.6 & $15.5-32$ & 21.6 & $12.5-31.2$ & 20.5 \\
\hline & 104B & 16.8 & $15.3-33$ & 21.7 & $12.4-26.5$ & 19.1 \\
\hline & $38 \mathrm{~B}$ & 16.9 & $12.5-26.5$ & 19.4 & $14.6-30$ & 20.1 \\
\hline \multirow[t]{3}{*}{ Volume weight } & 103B & 3.23 & $2.1-4.8$ & 3.9 & $3.6-5.6$ & 4.5 \\
\hline & 104B & 3.69 & $3-7.6$ & 4.9 & $3-5.4$ & 4.9 \\
\hline & $38 \mathrm{~B}$ & 3.61 & $3.1-8$ & 5.0 & $3.2-5.4$ & 4.4 \\
\hline
\end{tabular}

\begin{tabular}{|c|c|c|c|c|c|c|}
\hline \multirow[t]{2}{*}{ Traits } & \multirow[t]{2}{*}{ Crosses } & \multirow{2}{*}{$\begin{array}{c}\text { Parents } \\
\text { Mean }\end{array}$} & \multicolumn{2}{|c|}{$\begin{array}{l}\text { Backcross progenies } \\
\qquad\left(\mathrm{BC}_{2} \mathrm{~F}_{3}\right)\end{array}$} & \multicolumn{2}{|c|}{ Selfed progenies $\left(\mathrm{BC}_{1} \mathrm{~F}_{4}\right)$} \\
\hline & & & Range & Mean & Range & Mean \\
\hline \multirow[t]{3}{*}{ Oil content } & 103B & 32.26 & $25.1-39.8$ & 32.37 & $25.7-34.7$ & 30.82 \\
\hline & 104B & 36.60 & 23.9-39.6 & 32.40 & $25.6-39.3$ & 30.20 \\
\hline & $38 \mathrm{~B}$ & 33.49 & $23.4-37.1$ & 32.40 & $26.5-32.7$ & 28.80 \\
\hline \multirow{3}{*}{$\begin{array}{l}\text { Days to } 50 \text { per cent } \\
\text { flowering }\end{array}$} & $103 \mathrm{~B}$ & 60 & $51-60$ & 54 & $63-71$ & 67 \\
\hline & 104B & 58 & $57-62$ & 60 & $65-72$ & 68 \\
\hline & $38 \mathrm{~B}$ & 59 & $56-61$ & 59 & $56-71$ & 68 \\
\hline \multirow[t]{3}{*}{ Days to maturity } & 103B & 89 & $84-93$ & 88 & $98-112$ & 106 \\
\hline & 104B & 86 & $82-100$ & 92 & $100-113$ & 103 \\
\hline & $38 \mathrm{~B}$ & 88 & $92-100$ & 96 & $96-110$ & 102 \\
\hline
\end{tabular}


Similar results were line with Encheva and Christov (2006) showed intermediacy with regard to head diameter in interspecific cross between $H$. annuus (hybrid Albena) $\times H$. salicifolius and also observed that positive transgression was present for diameter. Hristovaet al., (2011) reported intermediate type of head diameter in the $\mathrm{F} 1$ hybrid derived between $\mathrm{H}$. annuus $\times H$. maximiliani. Prabakaran and Sujatha (2004) reported intermediate head type for the interspecific hybrids developed from $\mathrm{H}$. annuиs $\times H$. simulans.

The test weight of parental lines was an average of 5.2 grams. The backcross progenies showed intermediate test weight to both parental CMS lines and wild accessions ie. 4.1-4.5 g. while in selfed progenies recorded test weight were similar to their wild accessions (2.9-3.6 g) as reported by Vishnutej et al., (2016), Higher variability was observed for test weight in backcross progenies of 103B, 104B and 38B crosses in comparison with their selfed progenies. Similar results were found with Dudhe (2012) reported hybrid derive from cross between ARM 243 A $\times$ RHA-6D-1 showed intermediate seed weight in $\mathrm{F} 1$ hybrids. Hristova and Cherbadzi (2004) studied interspecific crosses involving annual diploid $H$. bolanderi Gray, H. neglectus Heiser and $H$. petiolaris having intermediate test weight, these results are also in accordance with the results obtained by Whelan and Dorrell (1980) for 100 seed weight in the interspecific derived hybrid between $H$. annuus $\times H$. maximiliani with an intermediate test weight.

Seed yield per plant is less in wild accessions as compared to cultivated lines (Prashanth et al., 2014) parental CMS lines had an average yield per plant of 16.39 g. Backcross progenies showed yield per plant in the range of 15.8-22.8 g. Backcross progenies had higher yield per plant than both the parents.
There is transgressive segregants appeared for this trait in backcross progenies. Higher variability was found in 103B, 104B and 38B crosses of backcross progenies compare to their respective selfed progenies. Results were on par with Nikolova and Christov (2004) for F1's derived from cross between $H$. annuus $\times$ $H$. laevigatas, reported high seed yield in the interspecific F1 hybrids. While in selfed progenies yield were lesser than parental CMS lines (11.1-13.6 g). But yield is not a simple character as it involves contribution more than one characters towards it based on the different yield contributing characters. Similar results were observed with Aswini (2018) reported that lesser seed yield in interspecific hybrids than female cultivated lines.

The oil content of the female parental CMS lines were recorded 34.11 per cent, In case of backcross progenies oil content was estimated as 32.37 to 32.4 per cent. While in selfed progenies oil content varied from 29.8 to 30.8 per cent. Backcross progenies 103B and 38B crosses exhibited higher variability for oil content as compare to their selfed progenies. While in 104B crosses both backcross and selfed progenies varied in a similar fashion. Comparatively backcross progenies had higher oil content than selfed progenies. This indicates the possibility of improving hybrid performance in backcross progenies more prominent than selfed progenies through further breeding and selection process for oil content. The results were contradictory with the results obtained by Whelan (1978) where he reported high oil content in the interspecific hybrids derived from cross between $H$. annuus and $H$. maximiliani. The performance of interspecific hybrids was either superior or nearer to their parents in respect of all yield contributing traits.

There is considerable difference in days to 50 per cent flowering. The parental CMS lines 
flowered early in 59 days. The backcross progenies showed 50 per cent flowering in the range of 54 to 60 days. While in selfed progenies days to 50 per cent flowering recorded from 67-68 days which indicates intermediacy in flowering in comparison to both parents. With respect to flowering duration backcross progenies of 103B, 104B and $38 \mathrm{~B}$ crosses were early types as compare to their respective selfed progenies. The results were on par with Vishnutej et al., (2016) interspecific crosses showed intermediacy in days to $50 \%$ flowering in comparison their parents. Meena et al., (2017) reported similar results in interspecific cross between $H$. annuus $\times H$. argophyllus. The cultivated species inbred ARM243B flowered early (48.5 days) while the $H$. argophyllus was late in flowering (79.4 days), the $\mathrm{F}_{1}$ was intermediate and flowered in 74.6 days. The 103B backcross progenies showed early flowering than female parent these result were in accordance with Encheva and Christov (2006) results showed that in the hybrid progenies of the interspecific cross $H$. annuus (hybrid Albena) $\times$ H. salicifolius recorded two to three days earlier flowering than that of the female parents. Results obtained by Atlagic (1996) for days to 50 per cent flower in the interspecific derived hybrid between $H$. annuus and $H$. occidentalis were late in flowering compared to the parent.

There exists much difference in case of days to maturity in between cultivated sunflower and wild annual diploid $H$. argophyllus (Meena et al., 2017). The parental CMS lines matured in 87 days. But backcross progenies were matured in 88-96 days which implies intermediate duration to both parents. While selfed progenies took 103-106 days for maturity which indicates the lateness of the crosses over female parent. Backcross progenies of 103B, 104B and 38B crosses matured early as compare to their selfed progenies. These results are in accordance with Prashanth et al., (2014) in which the interspecific hybrids $(\mathrm{M}-106 \times$ OCC 52and M-106 $\times$ MAX 1631) were late (90.4 and 95.4 days) in maturity compared to female parent (76.7 days), Similar results were reported by Hristova et al., (2011). They reported that interspecific hybrids of $H$. annuus $\times H$. maximiliani had more days to maturity. Nikolova and Christov (2004) reported similar results of days to maturity in 110-125 days in interspecific cross between H. annuus L. line LHA-300 x H.argophyllus (E-091).

In conclusion the mean performance and spectrum of genetic variation helps to identify superior crosses among backcross $\left(\mathrm{BC}_{2} \mathrm{~F}_{3}\right)$ and selfed progenies $\left(\mathrm{BC}_{1} \mathrm{~F}_{4}\right)$. The cross or families with high mean and variability could be effectively utilized to identify superior segregates.

The study revealed that variability created in head diameter, test weight, seed yield per plant, days to 50 per cent flowering and days to maturity were at desirable direction in backcross progenies ie., the mean of all these traits were shown higher end of the range as compare to their selfed progenies. Earlier reports also confirmed that these traits were utilized for sunflower crop improvement by the earlier findings of Nikolova and christov(2004), Encheva and Christov (2006), Hristova et al., (2011), Dudhe (2012), Prashanth et al., (2014), Vishnutej et al., (2016) and Meena et al., (2017) in prebreeding programmes. So that to create variability in these traits backcrossing is recommended as it reduces undesirable linkage drag rapidly as compared to advancement through selfing. It also gave a clear demarcation between backcross and selfed progenies derived from wide hybridization indicating the possibility to develop more stable and promising lines through two generation of backcrossing at 
early generation rather than progenies advancement only through selfing in sunflower pre-breeding programme.

\section{References}

Aswini, C., 2018, Cytomorphological characterization of intespecific hybrids in sunflower (Helianthus annuus L.). M.Sc. Thesis, Uni. Agric. sci. Raichur (India).

Atlagic, J. and Skoric, D., 1999, Cytogenetic study of Helianthus laevigatas and its $\mathrm{F}_{1}$ and $\mathrm{BC}_{1} \mathrm{~F}_{1}$ hybrids with cultivated sunflower (Helianthus annuus L.). Plant Breed., 118:555-559.

Atlagic, J., 1996, Cytogenetic studies in hexaploid Helianthus species and their $\mathrm{F}_{1}$ hybrids with cultivated Sunflower (Helianthus annuus L.). Plant Breed., 115: 257- 260.

Dudhe, M. Y., 2012, Hybrid purity assessment of sunflower hybrid by using molecular markers project. Paper presented at the international symposium on sunflower genetic resource. Turkey, $16-20^{\text {th }}$ October 2011, 34.

Encheva, J. and Christov, M., 2006, Interspecific hybrids between cultural sunflower (Helianthus annuus L.) and Helianthus salicifolius L. Helia,29(45): 107-116.

Hristova and Cherbadzi, M. M., 2004, Hybridization of cultural sunflower (Helianthus annuus L.) with the annual species Helianthus bolanderi Gray, Helianthus neglectus Heiser and Helianthus petiolaris Nuttall. Proc. 16th Int. Sunflower Conf., Fargo, ND, USA,699-707.

Hristova, C. M., Christov, M. and Valkova, D., 2011, Characteristics of some hybrid progenies produced by crossing of Helianthus annuus L. with Helianthus maximiliani Schrader.
Paper presented at the international symposium on sunflower genetic resource. Turkey, 29-42.

Jan, C. C., Feng, J., Seiler, G.J. and Gulya, T.J., 2006, Amphiploids of perennial Helianthus species $\mathrm{x}$ cultivated sunflower possess valuable genes for resistance to Sclerotinia stem and head rot. Proc. $28^{\text {th }}$ Sunflower Research Workshop, Fargo, ND, USA.

Meena H.P., Sujatha, M. and Prashant Kumar S., 2017, Interspecific hybrid between cultivated sunflower (Helianthus annuus L.) and silver leaf sunflower Helianthus argophyllus T. and G.: Cytomorphological and molecular characterization. Indian J. Genet., 77(4): 547-555.

Mohan, G. S., 2002, Studies on genetic enhancement of cultivated sunflower (Helianthus annuus L.) through interspecific hybridization. M.Sc. Thesis, Uni. Agri. sci. Banglore (India).

Nikolova, L. and Christov, M., 2004, Interspecific hybridization between Helianthus argophyllus and Helianthus annuus. Characteristics of some morphological and phytopathological traits in second and further hybrid generations. Proc. $16^{\text {th }}$ Int. Sunflower Conf., Fargo, ND USA, 731-736.

Nikolova, L., Christov, M., Nikolova, V., Shindrova, P. and Encheva, V., 1998, Interspecific hybridization between Helianthus annuus L. and Helianthus praecox spp. Hirtus Engleman and Gray. Helia, 21:15-22.

Prabakaran, A. J. and Sujatha, M., 2004, Interspecific hybrid of Helianthus annuus $\times$ Helianthus simulans, characterization and utilization in improvement of cultivated sunflower (Helianthus annuus L.). Euphytica, 135:275-282. 
Prashanth, Y., Praveen, K. G., Bharathi, M., Dudhe, Y. M. and Ramesh, M., 2014, Morphological characterization of interspecific hybrids between sunflower and wild Helianthus species Based on DUS Characters. Int. J. Appl. Bio. Pharmac. Tech., 5(1): 189194.

Sujatha, M., Prabakaran, A. J., Sangam, D. L., Chandra, S., 2008, Cytomorphological and molecular diversity in backcrossderived inbred lines of Sunflower (Helianthus annuus L.). Genome, 51(4): 282-293.

Terzic, S., Atlagic, J. and Pankovic, J., 2006, Characterization of $\mathrm{F}_{1}$ interspecific hybrids between wild Helianthus annuus L. Populations and cultivated sunflower. Genetica, 38(2): 159-168.

Valkova, D. and Christov, M., 2004, Characterization of F1 plants obtained from crosses between cultivated sunflower and wild annual Helianthus annuus. Proc. 16th Int. Sunflower Conf., Fargo, ND, USA, 747-750.

Vishnutej, E., Shankergoud, I. and Prabakaran, A. J., 2016, Morphological and molecular characterization of interspecific cross between cultivated sunflower (Helianthus annuus L.) with wild annual diploid Helianthus argophyllus. Elctron. J. Plant Breed., 7(2): 386-391.

Whelan, E. D. P. and Dorrell, D. G., 1980, Interspecific hybrids between Helianthus maximiliani Schrad and Helianthus annuus L. Effect of backcrossing on meiosis, anther morphology and seed characteristics. Crop Sci., 20: 29-34.

Whelan, E. D. P., 1978, Hybridization between annual and perennial diploid species of Helianthus. Canadian $J$. Genet. Cyto., 20: 523-530.

\section{How to cite this article:}

Sagar Iliger, V. V. Kulkarni, J. R. Diwan, S. Muniswami and Mallesh, S. B. 2020. Comparative Analysis of Backcross and Selfed Progenies in Sunflower (Helianthus annuus L.). Int.J.Curr.Microbiol.App.Sci. 9(09): 1764-1774. doi: https://doi.org/10.20546/ijcmas.2020.909.220 